\title{
Risk Modelling and Management: An Overview*
}

\author{
Chia-Lin Chang \\ Department of Applied Economics \\ Department of Finance \\ National Chung Hsing University \\ Taiwan \\ David E. Allen \\ School of Accounting, Finance and Economics \\ Edith Cowan University \\ Australia \\ Michael McAleer \\ Econometric Institute \\ Erasmus School of Economics \\ Erasmus University Rotterdam \\ and \\ Tinbergen Institute \\ The Netherlands \\ and \\ Department of Quantitative Economics \\ Complutense University of Madrid \\ Spain \\ and \\ Institute of Economic Research \\ Kyoto University \\ Japan \\ Teodosio Perez Amaral \\ Department of Quantitative Economics \\ Complutense University of Madrid \\ Spain
}

June 2013

* The authors are grateful to the numerous reviewers for their speed and efficiency, and to the Managing Editor and France Pinon for their assistance in preparing this volume. For financial support, the first author acknowledges the National Science Council, Taiwan, the second author thanks the Australian Research Council, and the third author is grateful to the Australian Research Council, the National Science Council, Taiwan, and the Japan Society for the Promotion of Science. 


\begin{abstract}
The papers in this special issue of Mathematics and Computers in Simulation are substantially revised versions of the papers that were presented at the 2011 Madrid International Conference on “Risk Modelling and Management” (RMM2011). The papers cover the following topics: currency hedging strategies using dynamic multivariate GARCH, risk management of risk under the Basel Accord: A Bayesian approach to forecasting value-at-risk of VIX futures, fast clustering of GARCH processes via Gaussian mixture models, GFC-robust risk management under the Basel Accord using extreme value methodologies, volatility spillovers from the Chinese stock market to economic neighbours, a detailed comparison of Value-at-Risk estimates, the dynamics of BRICS's country risk ratings and domestic stock markets, U.S. stock market and oil price, forecasting value-at-risk with a duration-based POT method, and extreme market risk and extreme value theory.
\end{abstract}

Keywords: Currency hedging strategies, Basel Accord, risk management, forecasting, VIX futures, fast clustering, mixture models, extreme value methodologies, volatility spillovers, Value-at-Risk, country risk ratings, BRICS, extreme market risk.

JEL: C14, C32, C53, C58, G11, G32. 


\section{Introduction}

The Madrid International Conference on "Risk Modelling and Management” (RMM2011) was held at the Real Centro Universitario (RCU) Escorial - María Cristina, San Lorenzo de El Escorial, Madrid, Spain, on 24 June 2011. RCU Escorial - Maria Cristina is part of the El Escorial Monastery, a Spanish royal site, which functions as a monastery, royal palace, museum, and school. El Escorial is also a UNESCO World Heritage Site. The conference was sponsored jointly by the International Association for Mathematics and Computers in Simulation (IMACS), Edith Cowan University, Australia, Complutense University of Madrid, Spain, and RCU Escorial - María Cristina., Spain.

The promotion, development, assistance and dissemination of knowledge in all areas of modelling and simulation are among the primary aims of IMACS. The society includes members with diverse backgrounds in a wide range of areas of professional interest. This interaction among disciplines, and the resulting cross-fertilisation of techniques and ideas, has traditionally been a prime objective of IMACS. The Madrid International Conference proved no exception, as illustrated by the diversity and innovative content of the selected papers in this special issue on risk modelling and management arising from the conference.

Conference participants included academics, post-doctoral students and graduate students. The opening session was addressed by Dean Agustín Alonso (RCU Escorial - Maria Cristina) and Robert Beauwens, President of IMACS.

The three sessions of the conference comprised 'Forecasting Value-at-Risk under the Basel Accord', 'Modelling Financial Portfolios', and 'Financial Econometrics'. Topical and interesting papers were presented on “A Detailed Comparison of Value-at-Risk Estimates”, "Extreme Market Risk - An Extreme Value Theory Approach”, “Forecasting Value-at-Risk with a Duration-based POT Method”, "Robust Risk Management Before, During and After the Global Financial Crisis: Further Results Based on Extreme Value Methodologies”, “Dynamics of Country Risk and Stock Market in the Five BRICS”, "Variance Clustering Improved Dynamic Conditional Correlation MGARCH Estimators”, "Risk Management of Risk Under the Basel Accord: A Bayesian Approach to Forecasting Value-atRisk of VIX Futures”, “Modelling Risk in Spot and Forward Exchange Rates”, "Bayesian Inference 
for Bivariate Markov-Switching Stochastic-Correlation Models", "Volatility Spillovers from the Chinese Stock Market to Economic Neighbours", and “The Rise and Fall of S\&P500 Variance Futures”.

There was substantial discussion after each presentation. The conference and dining facilities at RCU Escorial - María Cristina were excellent, and were enjoyed by all participants. A truly magnificent private tour of the El Escorial monastery was conducted by Dean Agustín Alonso at the end of the conference.

This special issue of Mathematics and Computers in Simulation contains substantially revised versions of the papers that were presented at the conference, illustrating the expertise and fields of research of expert modellers in Spain, Portugal, Italy, The Netherlands, Taiwan, Australia and the USA. Other participants in attendance were from various universities in Spain, Belgium, Italy, Germany and Taiwan.

\section{Overview}

This section presents an overview of the nine papers in this special issue of Mathematics and Computers in Simulation. Each of the nine selected papers in the special issue was fully reviewed by two referees, and represents a substantially edited, revised and/or extended version of what was presented at the conference. Papers in the special issue have been selected from a number of areas, namely currency hedging strategies using dynamic multivariate GARCH, risk management of risk under the Basel Accord: A Bayesian approach to forecasting value-at-risk of VIX futures, fast clustering of GARCH processes via Gaussian mixture models, GFC-robust risk management under the Basel Accord using extreme value methodologies, volatility spillovers from the Chinese stock market to economic neighbours, a detailed comparison of Value-at-Risk estimates, the dynamics of BRICS's country risk ratings and domestic stock markets, U.S. stock market and oil price, forecasting value-at-risk with a duration-based POT method, and extreme market risk and extreme value theory.

In the first paper, “Currency Hedging Strategies Using Dynamic Multivariate GARCH” [8], ChiaLin Chang (National Chung Hsing University, Taiwan), Lydia González-Serrano (Rey Juan Carlos 
University, Spain) and Juan-Angel Jimenez-Martin (Complutense University of Madrid, Spain) evaluate the effectiveness of using futures contracts as hedging instruments of the model of volatility used to estimate conditional variances and covariances, the analyzed currency, and the maturity of the futures contract being used. For this purpose, daily data of futures and spot exchange rates of three currencies, Euro, British pound and Japanese yen, against the American dollar are used to analyze hedge ratios and hedging effectiveness resulting from using two different maturity currency contracts, near-month and next-to-near-month contract. The authors estimate four well-known multivariate volatility models (namely CCC, VARMA-AGARCH, DCC and BEKK) and calculate optimal portfolio weights and optimal hedge ratios to identify appropriate currency hedging strategies. The hedging effectiveness index suggests that the best results in terms of reducing the variance of the portfolio are for the USD/GBP exchange rate. The empirical results show that futures hedging strategies are slightly more effective when the near-month futures contract is used for the USD/GBP and USD/JPY currencies. Moreover, the CCC and AGARCH models provide similar hedging effectiveness, although some differences appear when the DCC and BEKK models are used.

This is followed by the second paper, "Risk Management of Risk Under the Basel Accord: A Bayesian Approach to Forecasting Value-at-Risk of VIX Futures” [7], in which Roberto Casarin (Ca' Foscari University of Venice, Italy), Chia-Lin Chang (National Chung Hsing University, Taiwan), Juan-Angel Jimenez-Martin (Complutense University of Madrid, Spain), Michael McAleer (Erasmus University Rotterdam, The Netherlands) and Teodosio Perez Amaral (Complutense University of Madrid, Spain) evaluate the Basel II Accord and alternative forecasts of Value-at-Risk (VaR). It is well known that the Basel II Accord requires banks and other Authorized Deposit-taking Institutions (ADIs) to communicate their daily risk forecasts to the appropriate monetary authorities at the beginning of each trading day, using one or more risk models, whether individually or as combinations, to measure $\mathrm{VaR}$. The risk estimates of these models are used to determine capital requirements and associated capital costs of ADIs, depending in part on the number of previous violations, whereby realised losses exceed the estimated VaR. A new approach has been proposed in the literature for model selection to predict VaR, consisting of combining alternative risk models, and comparing conservative and aggressive strategies for choosing between VaR models. The authors address the question of risk management of risk, namely VaR of VIX futures prices, and extend the earlier approaches to examine how different risk management strategies performed during the 2008-09 global financial crisis (GFC). The empirical results suggest that an aggressive strategy 
of choosing the Supremum of single model forecasts, as compared with Bayesian and non-Bayesian combinations of models, is preferred to other alternatives, and is robust during the GFC. However, this strategy implies relatively high numbers of violations and accumulated losses, which are admissible under the Basel II Accord.

In the third paper, "Fast Clustering of GARCH Processes Via Gaussian Mixture Models” [2], Gian Piero Aielli (University of Padova, Italy) and Massimiliano Caporin (University of Padova, Italy) analyse multivariate conditional volatility using clustering methods. The financial econometrics literature includes several multivariate GARCH models where the model parameter matrices depend on a clustering of financial assets. Those classes might be defined a priori or may be data-driven. When the latter approach is followed, one method for deriving asset groups is given by the use of clustering methods. The authors analyze in detail a particular clustering approach, namely the Gaussian Mixture GARCH. This method is designed to identify groups based on the conditional variance dynamic coefficients. The clustering algorithm, based on a Gaussian Mixture model, has been recently proposed and is generalized with the introduction of a correction for the presence of correlation across assets. The authors introduce a benchmark estimator used to assess the performances of simpler and faster estimators. Simulation experiments show evidence of the improvements given by the correction for asset correlation.

The fourth paper is "GFC-robust Risk Management Under the Basel Accord Using Extreme Value Methodologies” [6], in which Paulo Araujo Santos (University of Lisbon, Portugal), Juan-Angel Jimenez-Martin (Complutense University of Madrid, Spain), Michael McAleer (Erasmus University Rotterdam, The Netherlands) and Teodosio Perez Amaral (Complutense University of Madrid, Spain) provide further evidence on the suitability of the median of the point Value-at-Risk (VaR) forecasts of a set of models as a Global Financial Crisis (GFC)-robust strategy by using an additional set of new extreme value forecasting models and by extending the sample period for comparison. These extreme value models include DPOT and Conditional EVT. Such models might be expected to be useful in explaining financial data, especially in the presence of extreme shocks that arise during a GFC. The empirical results confirm that the median remains GFC-robust even in the presence of these new extreme value models. This is illustrated by using the S\&P500 index before, during and after the 2008-09 GFC. The authors investigate the performance of a variety of single and combined $\mathrm{VaR}$ forecasts in terms of daily capital requirements and violation penalties under the Basel II 
Accord, as well as other criteria, including several tests for independence of the violations. The strategy based on the median, or more generally, on combined forecasts of single models, is straightforward to incorporate into existing computer software packages that are used by banks and other financial institutions.

In the fifth paper, "Volatility Spillovers from the Chinese Stock Market to Economic Neighbours" [3], David Allen (Edith Cowan University, Australia), Ron Amram (Edith Cowan University, Australia) and Michael McAleer (Erasmus University Rotterdam, The Netherlands) examine whether there is evidence of spillovers of volatility from the Chinese stock market to its neighbours and trading partners, including Australia, Hong Kong, Singapore, Japan and USA. China’s increasing integration into the global market may have important consequences for investors in related markets. In order to capture these potential effects, the authors explore these issues using an Autoregressive Moving Average (ARMA) return model. A univariate GARCH model is used to test for the persistence of volatility in stock market returns, as represented by stock market indices. Univariate GARCH, multivariate VARMA-GARCH, and multivariate VARMA-AGARCH models are also used to test for constant conditional correlations and volatility spillover effects across these markets. Each model is used to calculate the conditional volatility between both the Shenzhen and Shanghai Chinese markets and several other markets around the Pacific Basin Area, including Australia, Hong Kong, Japan, Taiwan and Singapore, during four distinct periods, beginning 27 August 1991 and ending 17 November 2010. The empirical results show some evidence of volatility spillovers across these markets in the pre-GFC periods, but there is little evidence of spillover effects from China to related markets during the GFC. This is presumably because the GFC was initially a US phenomenon, before spreading to developed markets around the globe, so that it was not a Chinese phenomenon.

The sixth paper is “A Detailed Comparison of Value at Risk Estimates” [1], in which Pilar Abad (Rey Juan Carlos University, Spain) and Sonia Benito (Universidad Nacional de Educación a Distancia, Spain) investigate the performance of different models of Value-at-Risk (VaR). The authors include several methods, namely parametric, historical simulation, Monte Carlo, and extreme value theory, and models to compute the conditional variance. They analyze several international stock indexes and examine two types of periods, namely stable and volatile periods. In order to choose the best model, the authors use a two-stage selection approach. The empirical results indicate 
that the best model is a parametric model with the conditional variance estimated with an asymmetric GARCH model under a Student t-distribution of returns. The paper shows that parametric models can obtain successful VaR estimates if the conditional variance is estimated properly.

In the seventh paper, “The Dynamics of BRICS’s Country Risk Ratings and Domestic Stock Markets, U.S. Stock Market and Oil Price” [9], Shawkat Hammoudeh (Drexel University, USA), Ramazan Sari (Middle East Technical University), Tengdong Liu (Drexel University, USA) and Mehmet Uzunkaya (TR Prime Ministry State Planning Organization) consider the country risk ratings of Brazil, Russia, India, China and South Africa (BRICS). These five countries are viewed currently as pillars of relative political, economic and financial stability, with the prospect of a major shift in future world power. The authors investigate the relationships among the economic, financial and political country risk ratings of the BRICS and relate those risk factors to their respective national stock markets in the presence of representatives of the world's major stock markets and oil market. They also examine the interrelationships among the national country financial risk ratings factors to determine the transmission of the risk spectrum among the countries of this group because of the relevance of this information to investors, traders and policy makers. The empirical results demonstrate that only the Chinese stock market is sensitive to all the factors. Financial risk ratings generally show greater sensitivity than economic and political risk ratings, and political risk is sensitive to both financial and economic risk ratings. Of the five BRICS, Brazil shows special sensitivity to economic and financial risks, while Russia and China hold strong sensitivity to political risk, and India demonstrates a special sensitivity to higher oil prices. Among the global factors, oil price is more sensitive to economic than financial risk, while the S\&P 500 index reverses this relationship. The two American quantitative easings affect BRICS differently.

In the penultimate paper, "Forecasting Value-at-Risk with a Duration-based POT Method” [5], Paulo Araujo Santos (University of Lisbon, Portugal) and Maria Isabel Fraga Alves (University of Lisbon, Portugal) analyse Value-at-Risk (VaR) using a different method. Threshold methods, based on fitting a stochastic model to the excesses over a threshold, are developed under the acronym POT (peaks over threshold). In order to eliminate the tendency to clustering of violations, the authors propose a model-based approach within the POT framework that uses the durations between excesses as covariates. Based on this approach, they suggest models for forecasting one-day ahead VaR. A simulation study is performed to validate the estimation procedure. Comparative studies with global 
stock market indices provide evidence that the proposed models can perform better than state-of-the art risk models and better than the widely used RiskMetrics model in terms of unconditional coverage, clustering of violations and capital requirements under the Basel II Accord.

The final paper is "Extreme Market Risk and Extreme Value Theory" [4], in which David Allen (Edith Cowan University, Australia), Robert Powell (Edith Cowan University, Australia) and Abhay Singh (Edith Cowan University, Australia) investigate extreme market risk. The occurrence of rare yet extreme events, also known as 'Black Swans', seems to be more apparent in financial markets around the globe. This means there is not only a need to design proper risk modelling techniques which can predict the probability of risky events in normal market conditions, but also a requirement for tools which can assess the probabilities of rare financial events, such as the recent Global Financial Crisis (2007-2008). An obvious candidate in dealing with extreme financial events and the quantification of extreme market risk is Extreme Value Theory (EVT). This proves to be a natural statistical modelling technique of relevance. EVT provides well established statistical models for the computation of extreme risk measures like the Return Level, Value-at-Risk (VaR) and Expected Shortfall. The authors apply Univariate Extreme Value Theory to model extreme market risk for the ASX All Ordinaries Australian index and the S\&P500 index. The authors demonstrate that EVT can be successfully applied to financial market return series for predicting static VaR, CVaR, Expected Shortfall (ES) and expected Return Level, and also daily VaR using a GARCH(1,1) model and EVTbased dynamic approach. 


\section{References}

[1] Abad, P. and S. Benito, A detailed comparison of value at risk estimates, to appear in Mathematics and Computers in Simulation, 2014.

[2] Aielli, G.P. and M. Caporin, Fast clustering of GARCH processes via Gaussian mixture models, to appear in Mathematics and Computers in Simulation, 2014.

[3] Allen, D.E., R. Amram and M. McAleer, Volatility spillovers from the Chinese stock market to economic neighbours, to appear in Mathematics and Computers in Simulation, 2014.

[4] Allen, D.E., R.J. Powell and A.K. Singh, Extreme market risk and extreme value theory, to appear in Mathematics and Computers in Simulation, 2014.

[5] Araujo Santos, P. and M.I. Fraga Alves, Forecasting value-at-risk with a duration-based POT method, to appear in Mathematics and Computers in Simulation, 2014.

[6] Araujo Santos, P., J.-A. Jimenez-Martin, M. McAleer and T. Perez Amaral, GFC-robust risk management under the Basel Accord using extreme value methodologies, to appear in Mathematics and Computers in Simulation, 2014.

[7] Casarin, R., C.-L. Chang, J.-A. Jimenez-Martin, M. McAleer and T. Perez Amaral, Risk management of risk under the Basel Accord: A Bayesian approach to forecasting value-at-risk of VIX futures, to appear in Mathematics and Computers in Simulation, 2014.

[8] Chang, C.-L., Lydia González-Serrano and J.-A. Jimenez-Martin, Currency hedging strategies using dynamic multivariate GARCH, to appear in Mathematics and Computers in Simulation, 2014.

[9] Hammoudeh, S., R. Sari, T. Liu and M. Uzunkaya, The dynamics of BRICS's country risk ratings and domestic stock markets, U.S. stock market and oil price, to appear in Mathematics and Computers in Simulation, 2014. 